\title{
A new and generic preparation method of mesoporous clay composites containing dispersed metal oxide nanoparticles
}

\author{
Jin Jun $\mathrm{Li}^{\mathrm{a}}$, Zhen $\mathrm{Mu}^{\mathrm{a}}$, Xiu Yan Xu ${ }^{\mathrm{a}}$, Hua Tian ${ }^{\mathrm{a}}$, Ming Hua Duan ${ }^{\mathrm{a}}$, Lan Dong $\mathrm{Li}^{\mathrm{a}}$, \\ Zheng Ping $\mathrm{Hao}^{\mathrm{a}, *}$, Shi Zhang Qiao ${ }^{\mathrm{b}, *}$, Gao Qing $\mathrm{Lu}^{\mathrm{b}}$ \\ ${ }^{a}$ Research Center for Eco-Environmental Sciences, Chinese Academy of Sciences, Beijing 100085, PR China \\ ${ }^{\mathrm{b}}$ ARC Centre for Functional Nanomaterials, School of Engineering and Australian Institute for Bioengineering and Nanotechnology, \\ The University of Queensland, Brisbane, QLD 4072, Australia
}

Received 23 October 2007; received in revised form 2 January 2008; accepted 4 January 2008

Available online 12 January 2008

\begin{abstract}
Nanosized oxide precursors of various transitional metals, including iron, chromium, cobalt, manganese and cerium, were prepared by dissolving their corresponding hydroxides in acetic acid solutions. These precursors were used to substitute the sodium ions in laponite clay like the way to prepare pillared clays, forming mesoporous solids with high surface areas of ca. $510-640 \mathrm{~m}^{2} / \mathrm{g}$. Stabilised by the adsorbed acetic acid molecules, the nanoparticle precursors of the metal oxides were well dispersed on the silicate platelets during the synthesis. The increase in the porosity after the intercalation of nanoparticles is primarily interpreted in terms of further delamination of the laponite clay caused by the reaction between the acidic metal precursor solutions and the silicate platelets. The clay composites containing nanoparticles of cobalt oxide appeared much more active than the impregnated cobalt catalysts in the deep oxidation of volatile organic compounds. (C) 2008 Elsevier Inc. All rights reserved.
\end{abstract}

Keywords: Mesoporous clay composites; Nanoparticles; Laponite; VOCs; Catalytic oxidation

\section{Introduction}

Nanosized particles of metal oxides are believed to be capable of exhibiting good catalytic behaviour in many heterogeneous catalytic reactions because of their high surface areas and specific surface properties [1-3]. However, nanoparticles are difficult to be preserved because they tend to agglomerate into large particles $[2,4]$. To obtain stable nano-catalysts, porous solids are often utilised as supporting media to disperse various nanoparticles of active phases $[2,3,5]$. At the same time, porous materials may provide high surface areas and easily accessible channels for reaction molecules and benefit their diffusion and catalytic reaction. Thus, the design of porous materials containing

\footnotetext{
* Corresponding authors. Tel.: +86 10 62849194; fax: +86 1062923564 (Z.P. Hao); tel.: +61 7 33463815; fax: +61 733656074 (S.Z. Qiao).

E-mail addresses: zpinghao@rcees.ac.cn (Z.P.Hao), s.qiao@uq.edu.au (S.Z. Qiao).
}

nanoparticles of functional metal oxides has attracted great interest in recent years [6].

Intercalated clay composites represent an important class of porous materials containing functional metal oxide nanoparticles on silicate layers [7-9], including pillared interlayered clays and delaminated derivatives. Such materials are prepared by exchanging the interlamellar alkali cations of certain laminated clays with particular metallic polyoxocations in aqueous solution, and subsequent calcinations convert these polyoxocations to robust nanoparticles of corresponding metal oxides anchored on the silicate platelets. In the structure of intercalated clays, the clay layers partition the metal oxide nanoparticles, preventing them from sintering. Their wide interlayer gallery can also prevent pores from being blocked by the metal oxide particles. Besides, there are some other merits about pillared clays for industrial applications, such as large surface areas, good thermal stability as well as special surface acidity $[7,8]$. 
In the synthesis of intercalated clays, the preparation of metal precursors is a crucial step. Up to now, in most cases partial hydrolysis of certain soluble salts in aqueous solutions at proper $\mathrm{pH}$ values is adopted to prepare polyoxocations as metal precursors [7-10]. However, only a limited number of transition metals such as iron, chromium, titanium and zirconium can form stable polycationic metal precursors by this way [7-10]. For other transition metal elements, it is difficult to produce stable partial hydrolysis products although they are very useful in catalysis. Some researchers have attempted to use polynuclear complexes as metal precursors [11], but the preparation processes of the polynuclear complexes were so complicated that they were rarely reported in the literatures [7-10]. Recently, Yurkov et al. [12] firstly synthesised interesting montmorillonite derivatives modified by nanosized cerium oxide with a high loading, in which $\left[\mathrm{Ce}_{6}(\mathrm{OH})_{12}\right]^{12+}$ polyoxocations were believed as precursors. However, to our knowledge, simple preparation methods for similar polyoxocations of other metals have not been reported. Therefore, a generic preparation method of metal precursors is desirable in the synthesis of clay composites containing the nanosized metal oxides.

In this paper, we present a new method for the preparation of various metal precursors, which involves dissolving metal hydroxides in acetic acid to obtain stable metal polycations. By this method, in addition to the extensively investigated iron and chromium, the nanosized particles of some other potentially active metals, such as manganese, cerium and cobalt, can be prepared, and used as metal precursors for fabricating metal-clay composites. Laponite, a type of commercial synthetic hectorite clay, was chosen as the host material. Laponite clay consists of small particles of ca. $25 \mathrm{~nm}$ in diameter, is dominated by delaminated structures [13], and has exchangeable hydrated sodium ions on its platelets to balance the negative surface charges $[8,14]$, thus is a desirable starting materials for fabricating nanomaterials $[15,16]$. Here we show that the various polycation precursors formed in acetic acid solutions can be used to produce mesoporous composites with high surface area and well-dispersed metal oxide nanoparticles on the silicate platelets. The synthesised cobalt oxide-clay composite was directly used as a catalyst in the deep oxidation of volatile organic compounds, which exhibited good catalytic property. Also, the developed mesoporosity of these clay derivatives make them desirable support materials for loading other catalytic active compositions. The novel method to prepare nanoparticle precursors demonstrated here may provide some new inspiration for the design of other nanomaterials.

\section{Experimental}

\subsection{Preparation of metal precursors}

To prepare precursors of various metals, the corresponding metallic hydroxides were firstly prepared by mixing $300 \mathrm{ml}$ of a solution containing $0.02 \mathrm{~mol}$ of metal salt with $100 \mathrm{ml}$ of a solution containing certain amount of sodium hydroxide. The metal salts used here were cobalt (II) sulfate, manganese (II) nitrate, cerium (III) nitrate, chromium (III) nitrate and iron (III) nitrate, and the corresponding molar ratios of metal to alkali were 1:2, 1:2, 1:3, $1: 3$, and 1:4, respectively. The fresh or aged hydroxides were dissolved in $150 \mathrm{ml}$ of an acetic acid solution under stirring, thus forming the metal precursors.

The traditional partial hydrolysis method was also used to prepare precursors of iron and chromium. In the case of iron, $0.01 \mathrm{~mol}$ of sodium carbonate was added slowly into $150 \mathrm{ml}$ of a solution containing $0.02 \mathrm{~mol}$ of iron (III) nitrate under continuous stirring, and then aged for $24 \mathrm{~h}$ at room temperature to form the traditional iron precursor. For chromium, $100 \mathrm{ml}$ of a solution containing $0.04 \mathrm{~mol}$ of sodium hydroxide was dropped slowly into $50 \mathrm{ml}$ of a solution containing $0.02 \mathrm{~mol}$ of chromium (III) nitrate under continuous stirring, and the traditional precursor of chromium was formed after ageing for $24 \mathrm{~h}$ at room temperature.

\subsection{Synthesis of metal oxide-clay composites}

Five grams of laponite was added to $250 \mathrm{ml}$ of distilled water, followed by stirring for $1 \mathrm{~h}$ to get a transparent dispersion. The solution of the metal precursor was added to this dispersion, keeping a constant metal to clay ratio of $4 \mathrm{mmol} / \mathrm{g}$. After continuous stirring for $1 \mathrm{~h}$, the mixture was aged at $308 \mathrm{~K}$ for more than $24 \mathrm{~h}$, then filtered and washed with deionised water repeatedly. The wet cake was dried at $373 \mathrm{~K}$ and calcined at $773 \mathrm{~K}$ in static air for three hours to obtain the metal oxide-clay composite products. The samples prepared using metal precursors in acetic acid solution were labelled as M-Lap $(\mathrm{M}=\mathrm{Co}, \mathrm{Ce}, \mathrm{Mn}$, $\mathrm{Cr}, \mathrm{Fe}$ ), while those prepared using traditional partially hydrolysed salts as precursors were denoted as M-LapB $(\mathrm{M}=\mathrm{Cr}, \mathrm{Fe})$.

In addition, alumina-clay composite (Al-Lap) was prepared according to our previous work [17], which can be used as desirable support material because it has a high specific surface area of ca. $550 \mathrm{~m}^{2} / \mathrm{g}$. Then cobalt nitrate was loaded on it by incipient wetness impregnation method to obtain supported cobalt oxide catalyst $\mathrm{Co}(w) / \mathrm{Al}$-Lap, where $w$ means the loading amount of cobalt.

\subsection{Characterizations of materials}

Transmission electron microscopy (TEM) images were collected on a Hitachi H-800 microscope operating at an accelerating voltage of $120 \mathrm{kV}$ for metal precursors and $150 \mathrm{kV}$ for metal oxide-clay composites.

Elemental analysis was performed on an EDAX energy dispersive X-ray spectroscopy micro-analyser which was coupled with a Hitachi S-3000N microscope.

Nitrogen adsorption-desorption isotherms at liquid nitrogen temperature were obtained on NOVA1200 gas sorption analyser. The samples were degassed under vacuum at $573 \mathrm{~K}$ for more than $6 \mathrm{~h}$ before the measurements. The BET surface areas were calculated based on the linear 
part of the BET plot $\left(P / P_{0}=0.05-0.25\right)$. The total pore volumes were estimated according to nitrogen uptake at a relative pressure $\left(P / P_{0}\right)$ of ca. 0.99 . The pore size distributions were derived from the adsorption branches of the isotherms using BJH method [18].

Powder X-ray diffraction (XRD) measurements were carried out on Siemens D5005 diffractometer at $40 \mathrm{kV}$ and $40 \mathrm{~mA}$ using a $\mathrm{Cu} \mathrm{K} \alpha$ radiation.

\subsection{Catalytic activity test}

Both the cobalt-containing clay composites prepared by the proposed method and the alumina-clay composite supported cobalt oxides were used as catalysts for the complete oxidation of benzene. The activity tests were performed in a continuous-flow fix-bed reactor. The catalysts were sieved to 40-60 meshes before loading in the reactor. In each test run, $400 \mathrm{mg}$ of catalyst was used, and the concentration of benzene was kept at ca. $1000 \mathrm{ppm}$, while the gas hourly space velocity was ca. $20,000 \mathrm{~h}^{-1}$. An on-line gas chromatography was used to analyse the concentration of benzene in the feed and effluent gas.

\section{Results and discussion}

\subsection{Formation of metal precursors}

In the traditional preparation method of ferric precursors, when a proper amount of sodium carbonate was added to a solution of ferric (III) nitrate under stirring with a $\mathrm{OH} / \mathrm{Fe}$ ratio of ca. 2.0, some stable partially hydrolysed products could be formed and the solution turned reddish brown gradually without the appearance of precipitates. These partially hydrolysed products can be used as metal precursors in the preparation of intercalated clays $[8,10]$. But when an excess amount of alkali was added to a solution of ferric (III) nitrate with a $\mathrm{OH} / \mathrm{Fe}$ ratio of ca. 4, precipitation of the gelatinous ferric hydroxide immediately appeared because of uncontrollable condensation of ferric species. The precipitates can be thoroughly dissolved in strong acid to form yellow brown solution containing ferric (III) ions again. However, it was found in our study that if the ferric hydroxide was dissolved in weak acetic acid, the resultant solution appeared to be reddish brown as the same as the colour of the partially hydrolysed ferric (III) solution promoted by alkali. Its TEM image reveals the presence of nano-sized particles of ferric species (Fig. 1a). Most of the particles are spherical in shape with a diameter of less than $4 \mathrm{~nm}$, while some are short rods. This indicates that it is difficult for the weak acid to break thoroughly all the $\mathrm{Fe}-\mathrm{O}$ bonds in the hydroxides to form simple ferric ions. The partial dissolution of the hydroxides in aqueous acetic solution resulted in the solids breaking into small particles of hydrated ferric oxide species, and some acetic acid molecules could adsorb on the surface of particles via strong interactions between the carboxyl group and the particles surface [19]. Acetic acid ligands could act as
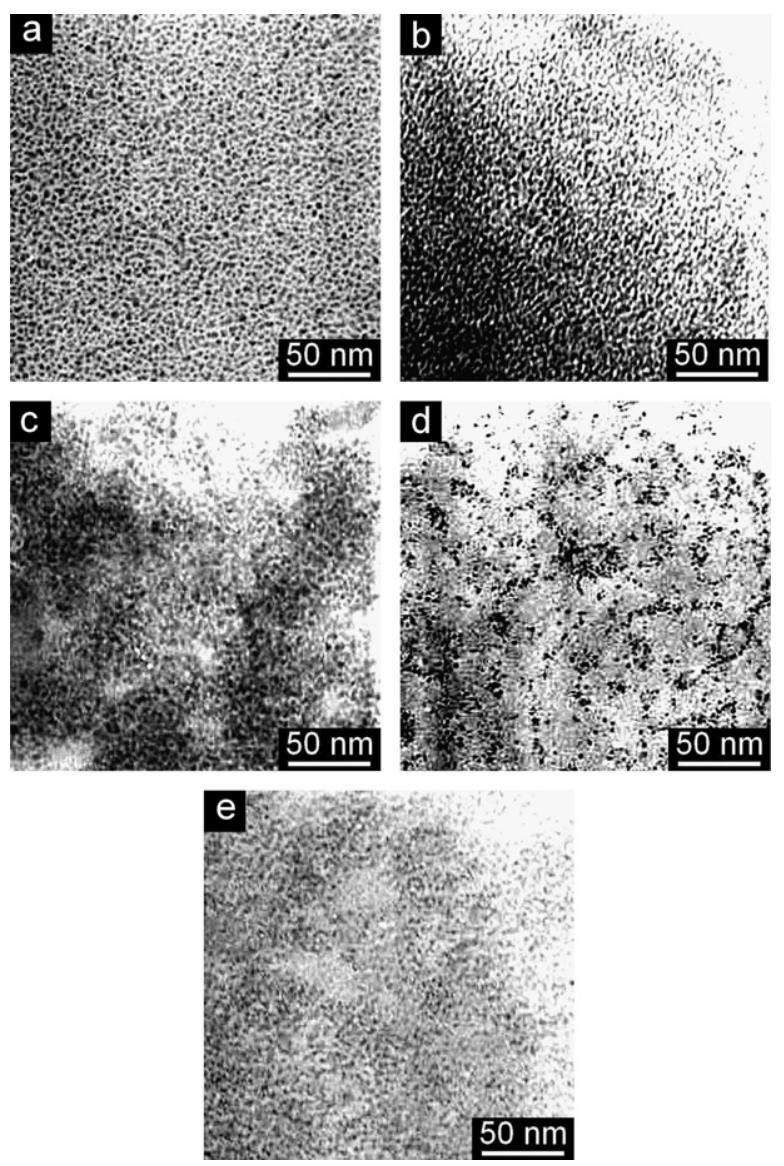

Fig. 1. TEM images of the nanosized metal precursors of (a) $\mathrm{Fe}$, (b) $\mathrm{Cr}$, (c) $\mathrm{Mn}$, (d) $\mathrm{Ce}$ and (e) $\mathrm{Co}$ in the acetic acid solutions.

stabilizing agents and prevent ferric precursors from aggregation in solution [20]. In the case of chromium, It was found that its freshly precipitated hydroxide can also be re-dissolved in acetic acid solutions to form nanosized polymeric species of chromium oxides (Fig. 1b).

Regarding other metallic elements, such as manganese, cerium and cobalt, their polycationic precursors cannot be prepared by the conventional partial hydrolysis method, since the addition of even a little amount of alkali would lead to the quick precipitation of their hydroxides. Nevertheless, it is easy to prepare their stable polymeric species by the new method. Dissolution of the freshly precipitated manganese (II) hydroxide in acetic acid yielded a solution with dark brown colour. Since manganese (II) is easily oxidised in an alkaline medium, manganese (II) hydroxide is immediately converted to dark brown hydrated manganese (IV) dioxide $\mathrm{MnO}(\mathrm{OH})_{2}$ [21], and the expected white manganese (II) hydroxide $\mathrm{Mn}(\mathrm{OH})_{2}$ was not observed even at the initial stage in our experiments. It is known that the stable form of manganese under acidic condition is manganese (II) ion [21], and its solution appears pale pink or nearly colourless. Thus, the dark brown colour in our experiment might arise from the dispersed nanoparticles of hydrated manganese (IV) oxide species formed by the partially dissolving of the precipitate. These nanoparticles can be clearly observed by TEM image in Fig. 1c. 
Table 1

Structural, composition and synthesis parameters of the clay composites

\begin{tabular}{|c|c|c|c|c|c|c|c|c|c|}
\hline Sample & $\begin{array}{l}\text { BET surface area } \\
\left(\mathrm{m}^{2} / \mathrm{g}\right)\end{array}$ & $\begin{array}{l}\text { Total pore volume } \\
\left(\mathrm{cm}^{3} / \mathrm{g}\right)\end{array}$ & $\begin{array}{l}\text { Average pore diameter } \\
(\mathrm{nm})\end{array}$ & $\mathrm{Mg} / \mathrm{Si}^{\mathrm{a}}$ & $\mathrm{Na} / \mathrm{Si}^{\mathrm{a}}$ & $\mathrm{M} / \mathrm{Si}^{\mathrm{a}}$ & $\mathrm{Wt}(\%)^{\mathrm{b}}$ & $\mathrm{pH}_{1}{ }^{\mathrm{c}}$ & $\mathrm{pH}_{2}{ }^{\mathrm{d}}$ \\
\hline Laponite & 338 & 0.25 & 2.9 & 0.666 & 0.093 & & & & \\
\hline Co-Lap & 639 & 0.48 & 3 & 0.282 & $-^{\mathrm{e}}$ & 0.024 & 1.98 & 2.5 & 3 \\
\hline Mn-Lap & 595 & 0.49 & 3.3 & 0.305 & - & 0.012 & 1.05 & 2.5 & 3.3 \\
\hline Ce-Lap & 594 & 0.46 & 3.1 & 0.245 & - & 0.017 & 3.34 & 2 & 2.7 \\
\hline Cr-Lap & 625 & 0.51 & 3.2 & 0.211 & - & 0.016 & 1.23 & 1.2 & 2.4 \\
\hline Cr-LapB & 460 & 0.34 & 3 & 0.543 & - & 0.401 & 20.71 & 3.7 & 4.3 \\
\hline Fe-Lap & 510 & 0.43 & 3.4 & 0.488 & - & 0.049 & 3.5 & 1.4 & 2.4 \\
\hline Fe-LapB & 451 & 0.32 & 2.8 & 0.596 & - & 0.405 & 22.86 & 1.9 & 2.1 \\
\hline
\end{tabular}

${ }^{\text {a }}$ Atomic ratio.

${ }^{b}$ Weight percent of the extrinsic metals in the clay composites.

${ }^{\mathrm{c}} \mathrm{pH}$ value of the metal precursor solutions.

${ }^{d} \mathrm{pH}$ value of the reaction mixture containing both the host laponite clay and the metal precursors.

e Sodium is not detectable.

The preparation of hydrated cerium oxide might also be accompanied with a change in the chemical valence. With the addition of alkaline to cerium (III) solution, yellow gelatinous hydrated ceria $\mathrm{CeO}_{2} \cdot n \mathrm{H}_{2} \mathrm{O}$ was immediately formed because white cerium (III) hydroxide can be oxidised in air [21]. The dissolution of the precipitates in acetic acid solution results in a yellow solution, and TEM image in Fig. 1d reveals that there exist even smaller particulate ceria species with diameters being mostly less than $3 \mathrm{~nm}$.

It should be emphasised that the freshly precipitated hydroxides of the above-mentioned metal elements must be separated immediately, and then acetic acid should be added quickly, otherwise the precipitate cannot be thoroughly re-dissolved to form stable dispersions. A possible explanation is that their hydroxides took phase changes so quickly that they transformed into rigid dense oxides after ageing for a while, which were less reactive and difficult to break into small clusters in acetic acid. However, the case is quite different for cobalt. The cobalt hydroxide in the slurry must be stirred until a colour change from blue to gray green was observed, then was separated and dissolved in acetic acid solution to form a stable dark red dispersion. Otherwise, only a red solution of cobalt (II) acetate can be obtained. Because cobalt (II) hydroxide can be slowly oxidised to cobalt (III) by the air [21], this dispersion might contain hydrated cobalt (III) oxide species of small sizes, which appears black colour. Some cobalt (II) ions might also exist in this dispersion because the cobalt (II) hydroxide was somewhat stable and could not be completely oxidised in the experiment condition. The dispersion appears dark red because of the co-presence of the two different forms of cobalt species. After reacting with the laponite dispersion, the cobalt oxide nanoparticles were incorporated into the laponite clay, forming a black precipitate, while leaving a red supernatant.

\subsection{Nanoparticles deposited on silicate platelets}

In laponite, sodium ions act as counterions to balance the negative charges on the clay layers. But in the prepared composites, sodium was not detected out as shown in Table 1 , and it appeared to be replaced by extrinsic metals. Fig. 2 shows the TEM images of the iron-containing composites prepared by both the new method and the traditional partial hydrolysis method. Apparently, the new method yielded product with finely dispersed particles on the clay layers (small dark dots on the silicate planes in Fig. 2a), and such particles are even somewhat difficult to be distin-
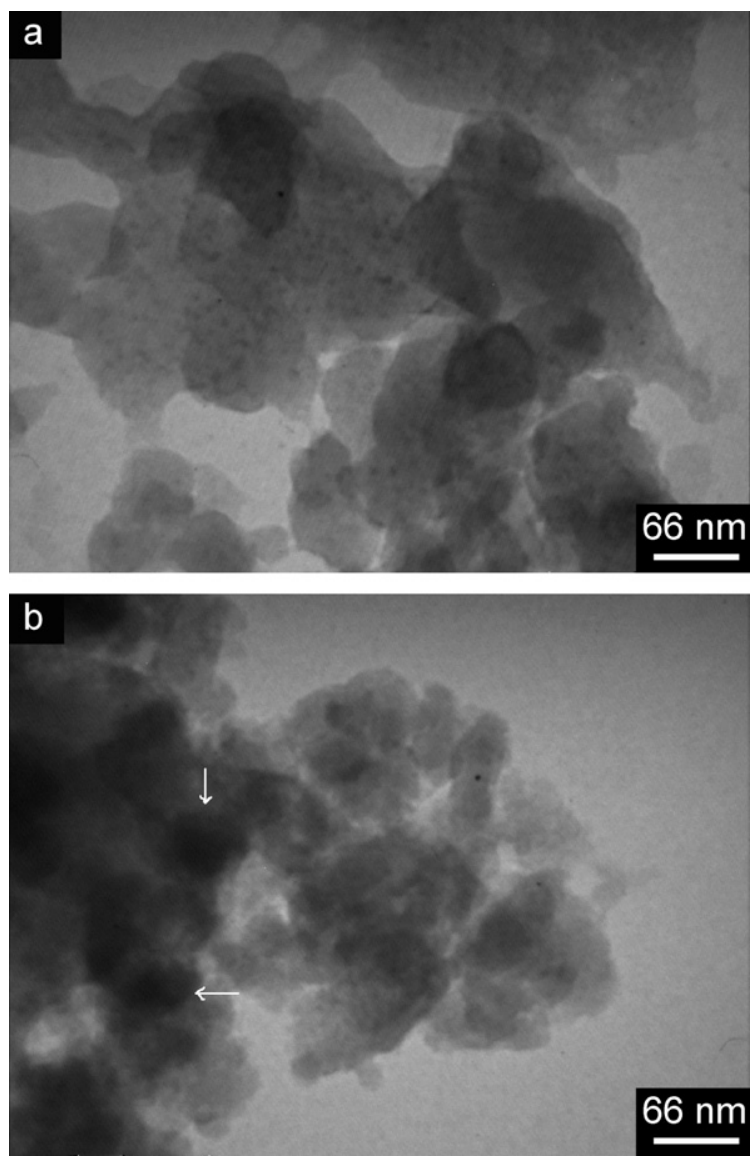

Fig. 2. TEM images of the iron-containing clay composites synthesised by (a) the new method and (b) the traditional method. 
guished in the TEM images of some other composites because of their tiny aspect. In contrast, the traditional method yielded product with severely aggregated oxide species on or beside the clay layers, as is proved in Fig. 2b (white arrows).

The dispersion of the incorporated metal oxides is also reflected by X-ray diffraction characterization (Fig. 3). For all the samples prepared by the new method, there are no additional diffraction peaks associated with the extrinsic metal oxides in their XRD patterns, indicating that the metal oxide species are finely dispersed on the clay platelets. In contrast, for the sample Cr-LapB prepared by traditional partial hydrolysis method, characteristic diffraction peaks of $\mathrm{Cr}_{2} \mathrm{O}_{3}$ can be clearly seen [22], which corroborates the existence of bulk oxide. This is consistent with TEM observations. No diffraction peaks of ferric oxides appear for Fe-LapB sample though bulk oxide has been observed in its TEM images as mentioned above, which indicates that the ferric oxide species may be poorly crystallised in this sample.

In the synthesis of the clay composites, the initial $\mathrm{pH}$ value of the laponite dispersion is ca. 10.3, and it will inevitably induce a rise in the $\mathrm{pH}$ value of the precursor solutions after mixing with each other (Table 1). Such increase in the $\mathrm{pH}$ value would promote the polymerization of the partially hydrolysed metal species [16,23], even

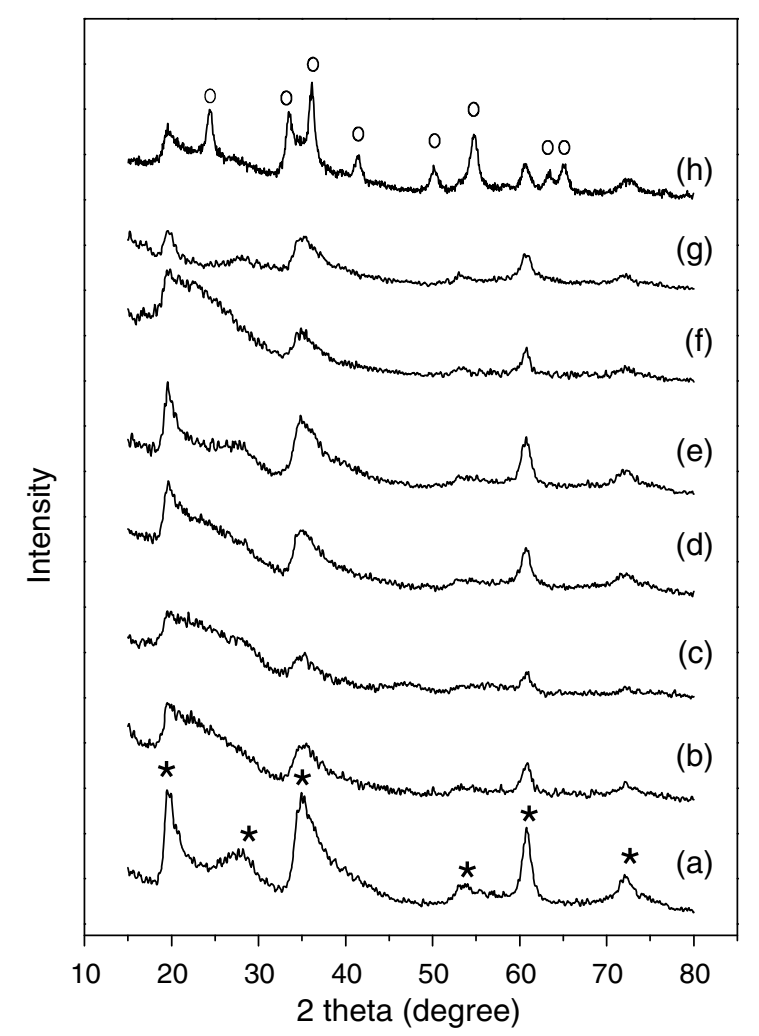

Fig. 3. XRD patterns of (a) the host laponite clay, the clay composites synthesised by the new method, including (b) Co-Lap, (c) Ce-Lap, (d) MnLap, (e) Fe-Lap, (f) Cr-Lap, and those prepared by the traditional method, including (g) Fe-LapB, (h) Cr-LapB. $\left(^{*}\right)$ is characteristic of hectorite, and $\circ$ is characteristic of $\mathrm{Cr}_{2} \mathrm{O}_{3}$. resulting in the formation of insoluble bulk hydroxide, i.e., the aggregation of partially hydrolysed metal species. On the other hand, it is known that the laponite particles in aqueous solution are stabilised by the negative surface charges [24], while the polyoxocations formed by partial hydrolysis are stabilized by positive charges. Thus, the mixing of the two dispersions will screen the electrostatic repulsion of each other, leading to the aggregation and precipitation of both substances. The aggregation of partially hydrolysed species was responsible for the high content of extrinsic metal in Fe-LapB and Cr-LapB as shown in Table 1. But the behaviours of the precursor particles in acetic acid solutions might be different. Some acetic acid molecules bonded to the precursor particles can probably serve as sheathing agent and prevent them from further condensation [20]. For instance, in the preparation of Fe-LapB, the $\mathrm{pH}$ value 2.1 in the final reaction mixture was just slightly higher than the $\mathrm{pH}$ value 1.9 in the initial precursor solution, but most of the ferric species precipitated after reaction, as evidenced by the nearly colourless supernatant. In contrast, in the preparation of Fe-Lap by new method, though the final mixture had an even higher $\mathrm{pH}$ value of 2.4, the supernatant still remained brown colour, which suggested that the surplus ferric species still existed in the solution instead of aggregating with each other. In other words, the new method can avoid the uncontrollable growth of metal oxide, producing well dispersed nanosized particles on the silicate platelets.

\subsection{Structural changes on the silicate platelets}

During the synthesis of intercalated clays, the leachingout of the atoms in the centre of octahedral sheet was always inevitable because of the acidic condition resulted from the metal precursor solutions $[15,16]$. The silicate platelet of laponite clays consists of an octahedral $\mathrm{MgO}_{6}$ sheet sandwiched between two tetrahedral $\mathrm{SiO}_{4}$ sheets $[14,25]$. After reacting with acidic metal precursors, the content of magnesium decreased (Table 1), indicating that a large number of magnesium atoms leached out as a result of the break of $\mathrm{Mg}-\mathrm{O}$ bond induced by protons. The decrease was more profound for those samples prepared by the new method. For example, the molar ratio of magnesium to silicon in sample Fe-Lap was lower than that in sample Fe-LapB (Table 1), though the former was synthesised at an even higher $\mathrm{pH}$ value. One possible reason is that acetate ions could chemically bond with magnesium atoms, forming complex compound, which promoted the dissolution of magnesium. In addition, protons might also be capable of attacking some $\mathrm{Si}-\mathrm{O}-\mathrm{Si}$ bonds in the tetrahedral sheets and bond with the unsaturated $\mathrm{Si}-\mathrm{O}$ units, leading to the formation of silanol groups in the framework of the silicate platelets. Besides, the ion exchange reactions between sodium ions and protons could also bring about silanol groups [14]. After calcination, some neighbouring silanol groups might condense to form amorphous silica structure $[16,26]$. From the XRD patterns of the clay composites 
(Fig. 3), we can see that typical characteristics of hectorite is still maintained [27]. Whereas, the peaks are less intense as compared to the host laponite clay. The peaks at $19.5^{\circ}$ and $28.2^{\circ}$ seem merged and broadened, which is probably a consequence of the overlap effect of clay and amorphous silica, as it has been reported that the amorphous silica phase formed upon magnesium dissolution corresponded to a broad peak between $20^{\circ}$ and $30^{\circ}$ [26]. Therefore, we can conclude that the final products consist of both hectorite clay and amorphous silica, which results in lower intensities in diffraction peaks of the XRD profiles.

\subsection{Textural evolution of the clay}

Fig. 4 displays the nitrogen adsorption-desorption isotherms and pore size distribution of iron oxide-clay composites prepared by two different methods. Both isotherms are of type IV [28], which corresponds to the trait
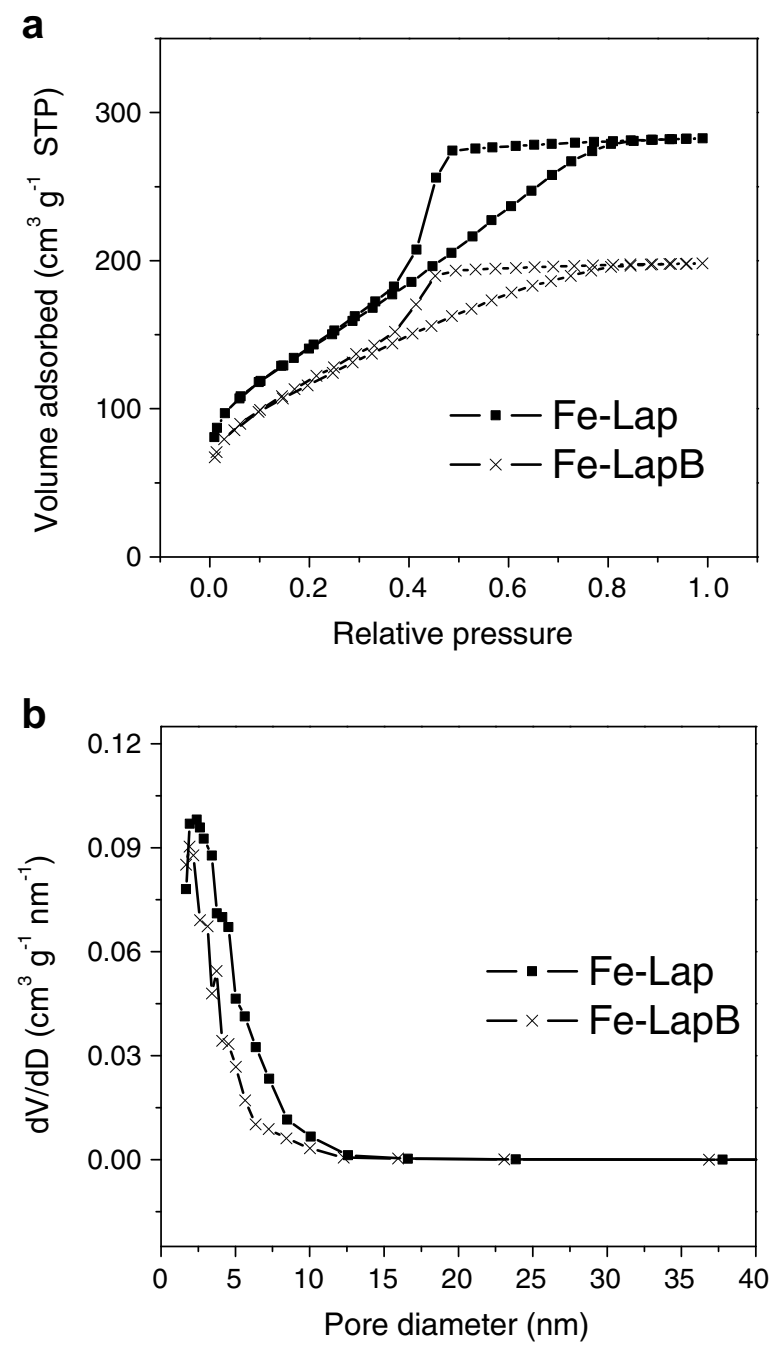

Fig. 4. (a) Nitrogen adsorption-desorption isotherms and (b) BJH pore size distributions of iron-containing clay composites synthesized by both traditional and new methods. of mesoporous materials. But Fe-Lap adsorbed apparently more nitrogen molecules than Fe-LapB (Fig. 4a), indicating that the new method produced larger pore volumes than the traditional method. Meanwhile, the former had larger pore size than the latter (Fig. $4 \mathrm{~b}$ and Table 1). Similar trend can be observed in the chromium oxide-clay composites. The features of the nitrogen adsorptiondesorption isotherms of other metal oxide-clay composites are similar to that of Fe-Lap.

Quantitative data of the textural properties derived from the nitrogen isotherms are shown in Table 1. Apparently, the composites have much higher surface areas and larger pore volumes than the host laponite clay, especially for those prepared using the new method. The specific surface area and total pore volume of the host laponite clay are $338 \mathrm{~m}^{2} / \mathrm{g}$ and $0.25 \mathrm{~cm}^{3} / \mathrm{g}$, respectively, while for the metal-clay composites prepared by the new method, they increase to ca. $510-640 \mathrm{~m}^{2} / \mathrm{g}$ and $0.43-0.51 \mathrm{~cm}^{3} / \mathrm{g}$, respectively. In contrast, the samples prepared using the traditional method have relatively lower surface area than $460 \mathrm{~m}^{2} / \mathrm{g}$ and smaller total pore volume than $0.34 \mathrm{~cm}^{3} / \mathrm{g}$. The concentrations of the acetic acid solutions for dissolving the hydroxides to form metal precursors affect the surface areas of the final products, as displayed in Fig. 5. To obtain metal oxide-clay composites with relatively higher surface areas, the proper acetic acid concentration to prepare the precursors of cobalt, cerium, manganese, chromium and iron should be ca. 28-67, 80, 40-60, 80 and $80-100 \mathrm{wt} \%$, respectively.

The preparation process of the metal oxide-clay composites involves the dispersion of the silicate platelets in water, the anchoring of the precursor particles on them,

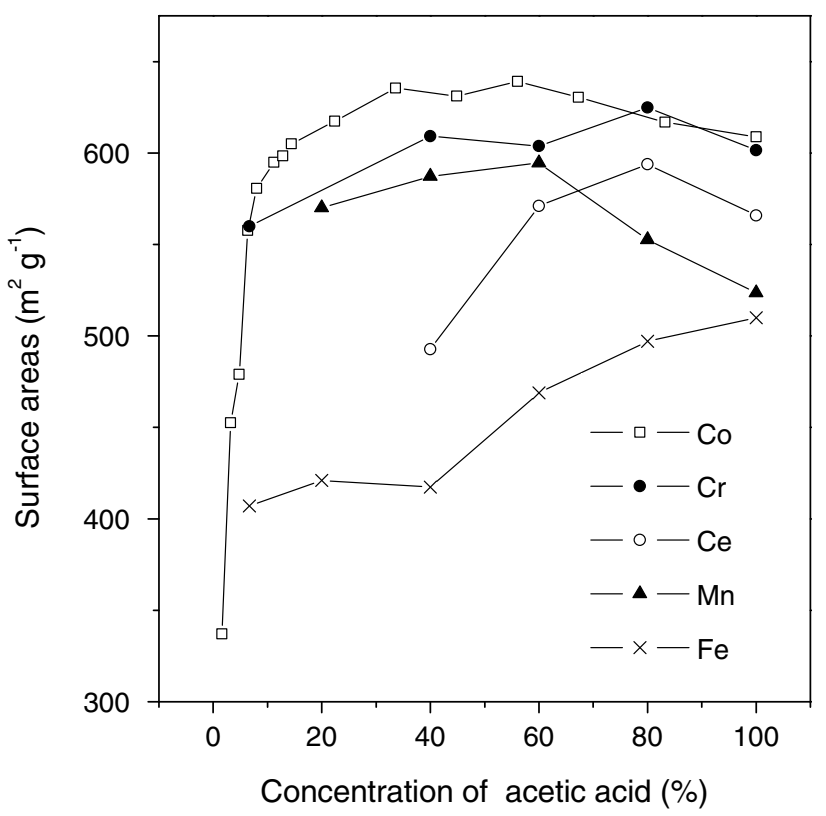

Fig. 5. The relation between the surface areas of the final composites and the concentrations of the acetic acid solutions to dissolving the hydroxides in preparing the metal precursors. 
and the flocculation of reaction mixture. The commercial laponite clay is composed of small platelets of about $25 \mathrm{~nm}$, and it can disperse in salt-free aqueous solution as individual particles and small oligomers, which are stabilized by the negative surface charge [24]. The addition of ionic metal precursors can induce flocculation of these particles by screening the electrostatic repulsion. The structural alterations and the adsorbing of precursor particles on the silicate platelets can induce different aggregation mode in the flocculated solids from the host clay. In the host laponite clay, face-to-edge and edge-to-edge association appear to be the main mode of silicate platelets aggregation, generally called delaminated clays, but this does not preclude the short-range face-to-face stacking in the interlayer direction [13]. The face-to-face association of silicate platelets is dominated by the van der Waals interaction between the neighbouring layers and the electrostatic attraction via the interlamellar alkali cations. However, as discussed above, the dissolution of part of the silicate framework could generate some silanol groups. Some of the silanol groups might stretch out of the plane of the silicate platelets, appearing like branches [14], as illustrated in Fig. 6. These irregular branches and the anchored precursor particles on the silicate planes would exhibit a steric effect in the flocculation step [14], which inhibited the face-to-face stacking of the platelets by reducing the interaction between the neighbouring layers. At the same time, the alkali cations were replaced by some large-sized extrinsic precursor particles in the reaction, which might also weaken interaction between the neighbouring layers. Consequently, the silicate platelets might show more house-ofcards aggregation in the products, namely, some originally short-range lamellar aggregated platelets could transfer to delaminated structure, as illustrated in Fig. 7. The surfaces in delaminated structure are more accessible for nitrogen molecules during the gas sorption experiments. Previous works have revealed that the delamination of clays could enhance significantly their surface areas as well as pore volumes [26]. In addition, because the laponite platelets are very small, the edge areas in this type of clay can contribute significantly to the total surface areas [25,29]. Thus the increased edge surfaces generated from the part breakdown of the clay platelets are also partially responsible for the enhancement of the specific surface areas.

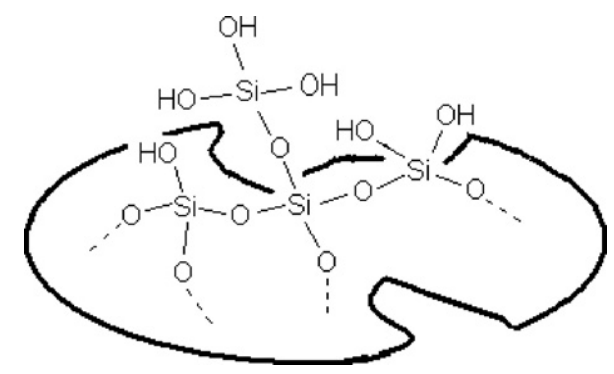

Fig. 6. Schematic illustration of the silicate platelets breakdown and the stretching-out of the silanol branches.

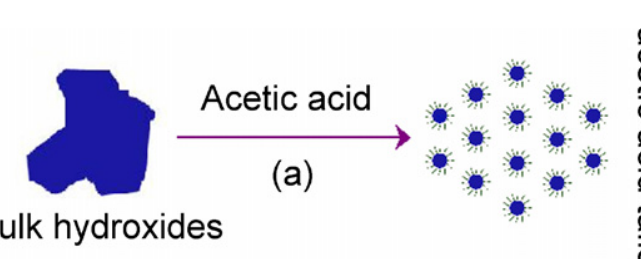

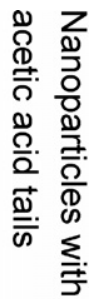

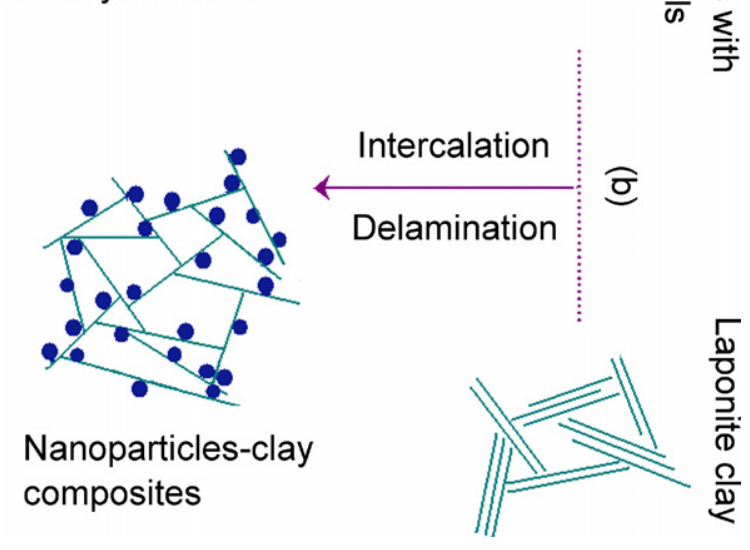

Fig. 7. Schematic illustration for the preparation of metal oxide nanoparticles-laponite clay composites. (a) Metal hydroxides breaking into small pieces with acetic acid molecule ligands; (b) incorporation of nanoparticles into the clay as well as further delamination of the clay structure.

\subsection{Catalytic performance in the deep oxidation of benzene}

Fig. 8 shows the ignition curves for the deep oxidation of benzene over clay composite catalysts containing cobalt oxide. Though the content of cobalt in Co-Lap is only ca. $2 \%$ (Table 1), its activity toward the oxidation of benzene is much more superior to the impregnated cobalt catalysts supported on alumina-clay composites with a loading of even up to $10 \%$. On Co-Lap, complete oxidation of benzene can be achieved at less than $573 \mathrm{~K}$, in contrast, on the other catalysts, complete conversion of benzene were not obtained even when the reaction temperature was raised to $673 \mathrm{~K}$. Impregnation is one of the most com-

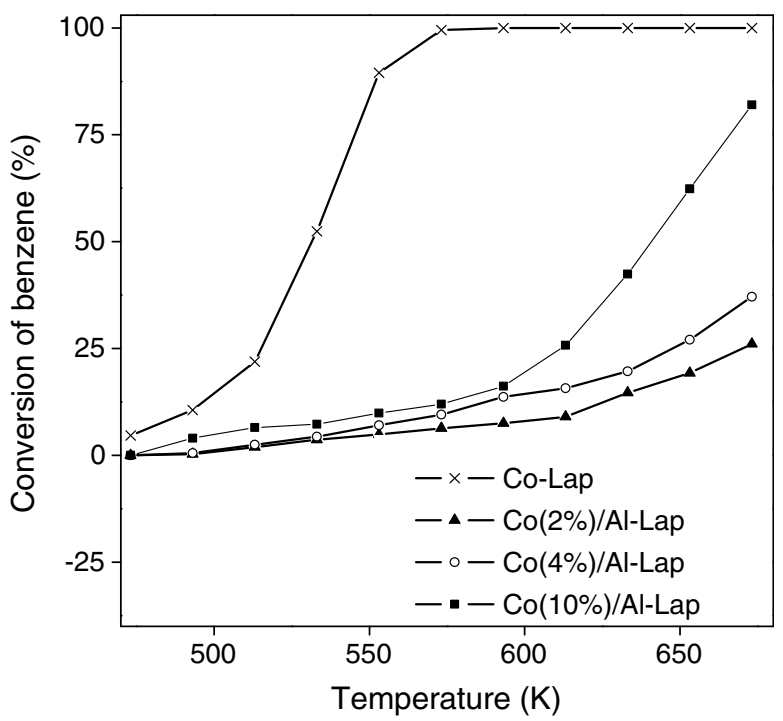

Fig. 8. Conversion curves of benzene over various cobalt-containing catalysts. 
monly used methods to prepare catalysts. However, this method has a drawback that metal oxides aggregates during the evaporation of impregnated solutions. In addition, the aggregated metal species can cause the blockage of the porosity of the support materials, exhibiting hindrance for the reaction molecules to approach the inner active phases [30]. In contrast, the growth of the metal oxides was effectively controlled in the synthesis of clay composites by new method, which insured a large active surface area or other special properties [1-3]. Moreover, the clay composites have developed mesoporosity, which favours the diffusion of reaction molecules. Thus the Co-Lap composite is fairly active in the deep oxidation of volatile organic compounds in comparison to the impregnated cobalt catalysts.

\section{Conclusion}

In this paper, we introduced an innovative approach to synthesize mesoporous clay compositions containing diverse well-dispersed transition metal oxides by using nanoparticles of hydroxide species as precursors, which were prepared by dissolving the corresponded metal hydroxides in aqueous solution of acetic acid. The prepared metal oxide-clay composites have surface areas of $510-640 \mathrm{~m}^{2} / \mathrm{g}$. The reaction between the acidic precursor solutions and the silicate platelets resulted in a more delaminated structure in the products, leading to the increase in the surface areas and pore volumes of the clay. Cobalt-containing clay composite prepared by the introduced method is more active than the impregnated cobalt catalysts in the deep oxidation of benzene. The fine dispersion of the nanoparticles and the developed mesoporosity of the composites are proposed to be responsible for its good catalytic behaviour. Furthermore, The method to prepare nanoparticle precursors might provide new opportunities for fabricating other nanomaterials for versatile applications.

\section{Acknowledgements}

This work was supported by the National Basic Research Program of China (No. 2004CB719500), the National High Technology Research and Development Program of China (2006AA06A310), and National Science Fund for Distinguished Young Scholars (20725723). Dr. J.J. Li appreciates the scientific research start fund in Chinese Academy of Sciences.

\section{References}

[1] G.A. Somorjai, Y.G. Borodko, Catal. Lett. 76 (2001) 1.

[2] A. Roucoux, J. Schulz, H. Patin, Chem. Rev. 102 (2002) 3757.

[3] C.R. Henry, Appl. Surf. Sci. 164 (2000) 252.

[4] L.S. Ott, R.G. Finke, Coord. Chem. Rev. 251 (2007) 1075.
[5] (a) D.T. On, D. Desplantier-Giscard1, C. Danumah, S. Kaliaguine, Appl. Catal. A 253 (2003) 545;

(b) J. Perez-Juste, I. Pastoriza-Santos, L.M. Liz-Marzan, P. Mulvaney, Coord. Chem. Rev. 249 (2005) 1870;

(c) B.C. Gates, Chem. Rev. 95 (1995) 511;

(d) A. Taguchi, F. Schuth, Micropor. Mesopor. Mater. 77 (2005) 1.

[6] (a) U.H. Lee, J.H. Lee, D.Y. Jung, Y.U. Kwon, Adv. Mater. 18 (2006) 2825;

(b) H. Song, R.M. Rioux, J.D. Hoefelmeyer, R. Komor, K. Niesz, M. Grass, P. Yang, G.A. Somorjai, J. Am. Chem. Soc. 128 (2006) 3027; (c) A. Wang, Y.P. Hsieh, Y.F. Chen, C.Y. Mou, J. Catal. 237 (2006) 197.

[7] A. Gil, L.M. Gandia, Catal. Rev. Sci. Eng. 42 (2000) 145.

[8] J.T. Kloprogge, J. Porous Mater. 5 (1998) 5.

[9] A.A.G. Tomlinson, J. Porous Mater. 5 (1998) 259.

[10] K. Ohtsuka, Chem. Mater. 9 (1997) 2039.

[11] (a) T. Mishra, K. Parida, Appl. Catal. A 174 (1998) 91;

(b) T. Mishra, K. Parida, J. Mater. Chem. 7 (1997) 147;

(c) S. Thomas, J.A. Bertrand, M.L. Occelli, Chem. Mater. 11 (1999) 184.

[12] L.V. Gorobinskii, G.Y. Yurkov, D.A. Baranov, Micropor. Mesopor. Mater. 100 (2007) 134.

[13] M.L. Occelli, J. Lynch, J. Catal. 107 (1987) 557.

[14] N.H. Tran, M.A. Wilson, A.S. Milev, G.R. Dennis, A.L. McCutcheon, G.S.K. Kannangara, R.N. Lamb, Langmuir 22 (2006) 6696.

[15] (a) H.Y. Zhu, Z.P. Hao, J.C. Barry, Chem. Commun. 23 (2002) 2858; (b) Z.P. Hao, H.Y. Zhu, G.Q. Lu, Appl. Catal. A 242 (2003) 275.

[16] H.Y. Zhu, J.C. Zhao, J.W. Liu, X.Z. Yang, Y.N. Shen, Chem. Mater. 18 (2006) 3993.

[17] J.J. Li, Z. Jiang, Z.P. Hao, X.Y. Xu, Y.H. Zhuang, J. Mol. Catal. A $225(2005) 173$.

[18] E.P. Barrett, L.G. Joyner, P.P. Halenda, J. Am. Chem. Soc. 73 (1951) 373.

[19] (a) L. Ojamäe, C. Aulin, H. Pedersen, P. Käll, J. Coll. Interf. Sci. 296 (2006) 71 ;

(b) C.Y. Wang, H. Groenzin, M.J. Shultz, J. Am. Chem. Soc. 127 (2005) 9736.

[20] (a) H.Y. Fan, K. Yang, D.M. Boye, T. Sigmon, K.J. Malloy, H.F. Xu, G.P. Lopez, C.J. Brinker, Science 304 (2004) 567;

(b) H. Bönnemann, R.M. Richards, Eur. J. Inorg. Chem. 2001 (2001) 2455;

(c) S. Panigrahi, S. Basu, S. Praharaj, S. Pande, S. Jana, A. Pal, S.K. Ghosh, T. Pal, J. Phys. Chem. C 111 (2007) 4596;

(d) J. Kim, J.E. Lee, J. Lee, J.H. Yu, B.C. Kim, K. An, Y. Hwang, C.H. Shin, J.G. Park, J. Kim, T. Hyeon, J. Am. Chem. Soc. 128 (2006) 688.

[21] X.Z. Cao, T.Y. Song, X.Q. Wang, Inorganic Chemistry, High Education Press, Beijing, 1994.

[22] L. Li, Z.F. Yan, G.Q. Lu, Z.H. Zhu, J. Phys. Chem. B 110 (2006) 178.

[23] J.L. Valverde, P. Sanchez, F. Dorado, C.B. Molina, A. Romero, Micropor. Mesopor. Mater. 54 (2002) 155.

[24] T. Nicolai, S. Cocard, Langmuir 16 (2000) 8189.

[25] J.M. Saunders, J.W. Goodwin, R.M. Richardson, B. Vincent, J. Phys. Chem. B 103 (1999) 9211.

[26] M.A. Vicente, M. Suarez, J.D.D. LopezGonzalez, Langmuir 12 (1996) 566.

[27] R.E. Grim, Clay Mineralogy, McGraw-Hill, New York, 1953.

[28] K.S.W. Sing, D.H. Evrett, R.A.W. Haul, L. Moscou, R.A. Pierotti, J. Rouqerol, T. Siemieniewska, Pure Appl. Chem. 57 (1985) 603.

[29] R.K. Kukkadapu, L. Kevan, J. Phys. Chem. 93 (1989) 1654.

[30] J.J. Li, X.Y. Xu, Z. Jiang, Z.P. Hao, C. Hu, Environ. Sci. Technol. 39 (2005) 1319. 